\title{
Immunization rates and timely administration in pre-school and school-aged children
}

Received: 12 May 2005 / Revised: 19 August 2005 / Accepted: 21 August 2005 / Published online: 3 December 2005

(C) Springer-Verlag 2005

\begin{abstract}
Whereas immunization coverage has been repeatedly assessed in the Swiss population, little is known about the timely administration of universally recommended immunizations in Switzerland and elsewhere. The goal of this study was to determine compliance with official standard immunization recommendations in preschool and school-aged children in Basel, Switzerland, focusing on coverage rates and timely administration. Of a cohort of children entering kindergarten and third-grade primary school in Basel in 2001, 310 and 310, respectively, were identified in proportion to the overall age-appropriate populations in the four city districts. Foreign-born children were excluded. The data were extracted from immunization records provided voluntarily by parents. Coverage for three doses of diphtheria, tetanus, and poliomyelitis vaccines was $>95 \%$ and $<90 \%$ for pertussis and Hib. The rates of age-appropriate booster doses were significantly lower, especially for pertussis and Hib $(<60 \%)$. Cumulative coverage for measles, mumps, and rubella (MMR) was $<90 \%$ for the first dose and $33 \%$ for the second dose by 10 years of age. All immunizations were administered with significant delays. Coverage for the first three doses of DTP combination vaccines did not reach $90 \%$ before 1 year of age and, for the first dose of MMR, a plateau just below $80 \%$ was not reached before 3 years of age. Delayed administration of immunizations in childhood, as well as complete lack of booster doses in a significant fraction of children, with important implications for public health have been discovered in this study. This may lead to fatal disease in individuals, epidemics in the community, and threatens
\end{abstract}

This work contains data from the medical thesis of Mirjam Zuberbühler at the Medical Faculty, University of Basel, Switzerland

U. Heininger $(\bowtie) \cdot M$. Zuberbühler

University Children's Hospital (UKBB),

P.O. Box, 4005 Basel, Switzerland

e-mail: Ulrich.Heininger@unibas.ch

Tel: $+41-61-6856565$

Fax: +41-61-6856012 national and international targets of disease elimination, such as measles and congenital rubella syndrome.

Keywords Immunization - Coverage $\cdot$ Children · Timely immunization

Abbreviations DTPa: Diphtheria, tetanus, acellular pertussis component - IPV: Inactivated poliomyelitis vaccine - OPV: Oral poliomyelitis vaccine -

Hib: Haemophilus influenzae type b .

MMR: Measles, mumps, rubella .

HBV: hepatitis B vaccine

\section{Introduction}

Compliance with recommended immunizations is usually estimated based on immunization coverage in specific age groups. These data, however, do not allow the assessment of timely immunization and, therefore, information on this is scarce in Switzerland and elsewhere $[1,4,5,8-10,12$, 14]. This is unfortunate, since knowledge on the extent of timely immunization, especially in children, would be useful for the improved control of vaccine-preventable diseases. If immunizations are administered with significant delays, individual as well as herd immunity is suboptimal and, not surprisingly, outbreaks of diseases such as pertussis or measles will occur $[11,13]$.

The current Swiss standard immunization plan in childhood comprises vaccinations against nine diseases, i.e., DTPa-IPV-Hib (at 2, 4, 6, and 15-24 months of age), MMR (12 and 15-24 months), DTPa-IPV (4-7 years), and Td and HBV (11-15 years) [16]. Immunization against Hib has been recommended since November 1991. The forth and fifth dose of pertussis vaccine have been introduced in 1996, as was a second dose for MMR (at 4-7 years, recently changed to 15-24 months). Immunizations in Switzerland are usually administered by pediatricians and general practitioners in private practices; in addition, catchup immunizations are frequently offered by the School Health Services in kindergarten and schools. Moreover, 
Fig. 1 Study flow chart

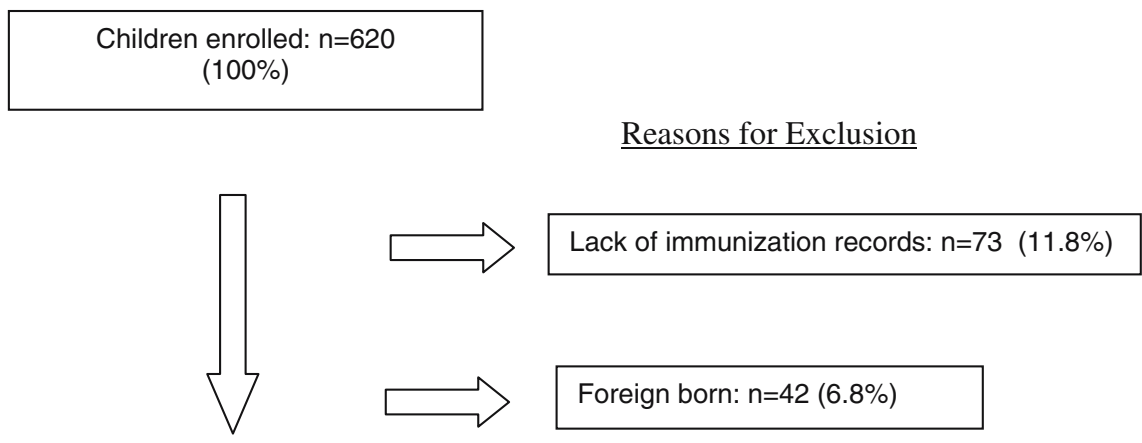

Included in final analysis: $\mathrm{n}=505$

$(81.5 \%)$

computer-based reminder systems are used by some physicians, but the extent of use of this procedure is unknown.

The goal of this prospective, exploratory cohort study was to estimate immunization coverage and timely administration in kindergarten and school-aged children in Basel, Switzerland, as a basis for targeted activities for improvement in the future.

\section{Methods}

Study cohort and data collection

As part of regular standard procedures performed by the local School Health Service, immunization records of all children admitted to the first year of kindergarten or third year of primary school are assessed on a voluntary basis in the Canton of Basel every year. Age-appropriate coverage is documented and individual recommendations for catchup immunizations are given to parents in written form. For the purpose of this study, performed in 2001, not only the numbers of specific immunizations, but also, each child's exact age at each immunization was recorded.
In the Canton of Basel, yearly assessment of immunization rates is performed in a pre-determined sequence of visits to all kindergarten and schools. The Canton is divided into four districts and the numbers of inhabitants and proportions of children are not equally distributed. To achieve a proportionate and representative study sample, a total of 310 kindergarten and 310 school children were selected from the four districts according to the overall age-appropriate distribution of children. The study size of 310 children per cohort was determined based on the following assumptions: a yearly cohort of approximately $1,500-1,600$ children entering the kindergarten/school system in Basel; a study participation rate (per protocol) of 50-60\%; at least $10 \%$ of all children residing in Basel were to be included in the study.

Study enrollment was done in a consecutive fashion until the pre-determined number of study subjects was reached. Children who were not born and had not lived in Switzerland when they were 2 months of age (and, therefore, were not necessarily immunized according to the Swiss schedule) and children without available immunization records were excluded from the per protocol analysis.

Table 1 Immunization coverage in the 505 study subjects

\begin{tabular}{lllllr}
\hline & $\geq 1$ dose, $N(\%)$ & $\geq 2$ doses, $N(\%)$ & $\geq 3$ doses, $N(\%)$ & $\geq 4$ doses, $N(\%)$ & $\geq 5$ doses, $N(\%)$ \\
\hline Diphtheria & $498(98.6)$ & $496(98.2)$ & $491(97.2)$ & $457(90.5)$ & $192(71.4)^{*}$ \\
Tetanus & $499(98.8)$ & $498(98.6)$ & $492(97.4)$ & $457(90.5)$ & $190(70.6)^{*}$ \\
Pertussis & $468(92.7)$ & $456(90.3)$ & $437(86.5)$ & $281(55.6)$ & $9(3.5)^{* *}$ \\
Hib*** & $292(96.1)$ & $286(94.1)$ & $265(87.2)$ & $155(51.0)$ & n.a. \\
Poliomyelitis & $499(98.8)$ & $498(98.6)$ & $488(96.6)$ & $431(85.3)$ & $158(58.7)^{*}$ \\
Measles & $449(88.9)$ & $135(50.2)^{*}$ & n.a. & n.a. & n.a. \\
Mumps & $443(87.7)$ & $135(50.2)^{*}$ & n.a. & n.a. & n.a. \\
Rubella & $440(87.1)$ & $135(50.2)^{*}$ & n.a. & n.a. & n.a. \\
\hline
\end{tabular}

n.a. not applicable

* Percentage refers to 269 children born before 1 January 1994 (i.e., $>7$ years of age during study period)

** Percentage refers to 258 children born between 1 January 1991 (i.e., eligible for the 5-dose recommendation established in 1996) and 31 December 1993 (i.e., $>7$ years of age during study period)

*** Percentage refers to 304 children born 1 January 1992 or later (i.e., eligible for Hib immunizations in infancy, recommended since 1991) 
Fig. 2 Cumulative vaccination coverage with diphtheria vaccine doses $1-3$ in 304 children with recommended time points at 2, 4, and 6 months of age. Diamonds dose 1. Squares dose 2. Triangles dose 3

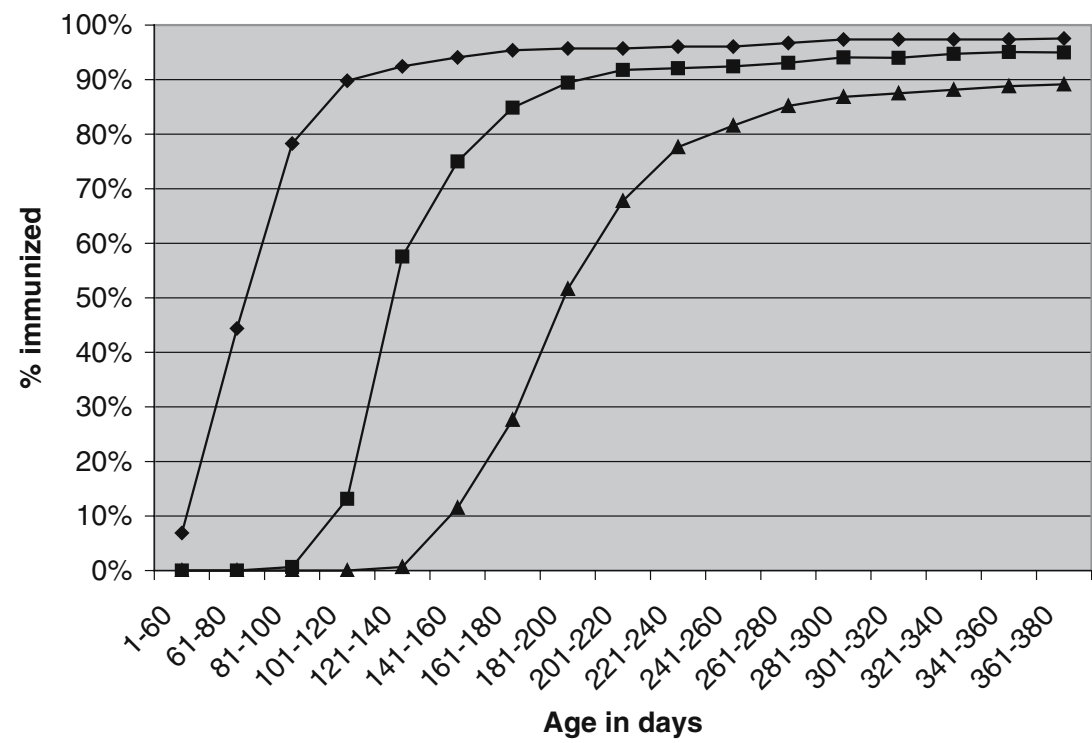

\section{Definitions}

In accordance with the Swiss national immunization recommendations, timely immunization was defined as follows:

- For doses 1-3 of DTPa (doses 1 and 2, if DT only), OPV or IPV, and Hib: administered with less than 4 weeks delay

- For dose 4 of DTPa (dose 3, if DT only), OPV or IPV, and Hib: administered before second birthday

- For 1st dose of MMR: administered before 24 months of age

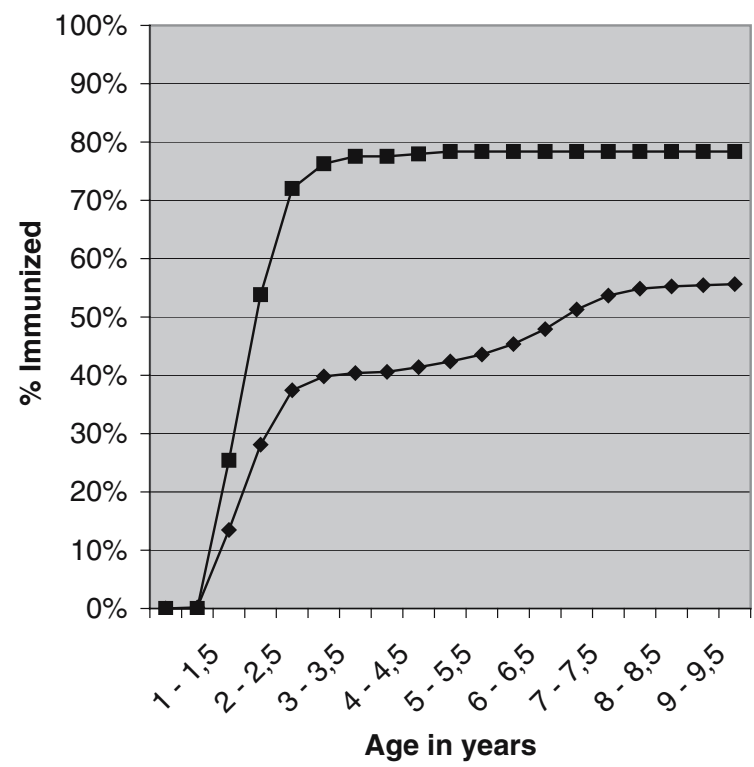

Fig. 3 Cumulative vaccination coverage with a forth dose of pertussis component vaccine in 236 children born in September 1994 or later, and in the total study cohort $(n=505)$. Squares children born September 1994 or later $(n=236)$. Diamonds all study children $(n=505)$
- For 2nd dose of MMR: administered before seventh birthday

Statistical analysis

Statistical analyses were performed by the use of SPSS version 10.0 (SPSS Inc., Chicago, USA).

\section{Ethical approval}

The study protocol was approved by the University of Basel Medical Faculty Ethical Committee. As a standard procedure, the parents were informed via letter about the purpose of the assessment of immunization status and were asked to bring their child's personal immunization records to kindergarten or school on a specific date for inspection by members of the School Health Service on a voluntary basis.

Children were considered to be "foreign born" if the first immunization in their first year of life had been administered outside Switzerland.

\section{Results}

Characteristics of the study participants

There were 1,625 children entering kindergarten and 1,518 children entering third grade primary school in Basel in 2001. The relative distributions in the four districts were $35.1 \%, 20.7 \%, 30.3 \%$, and $13.8 \%$ for the new kindergarten entrees and $35.0 \%, 22.2 \%, 28.2 \%$, and $14.6 \%$ for the third grade entrees. Accordingly, the first 109 (35.2\%), 64 $(20.6 \%), 94(30.3 \%)$, and $43(13.9 \%)$ of kindergarten children in the four respective districts were selected for study participation in chronological order. The respective figures for the third grade entrees were $109,68,88$, and 45 . Of the 620 children enrolled, 115 (18.6\%) were excluded 
Fig. 4 Cumulative vaccination coverage with one and two doses of measles containing vaccine in 505 study children

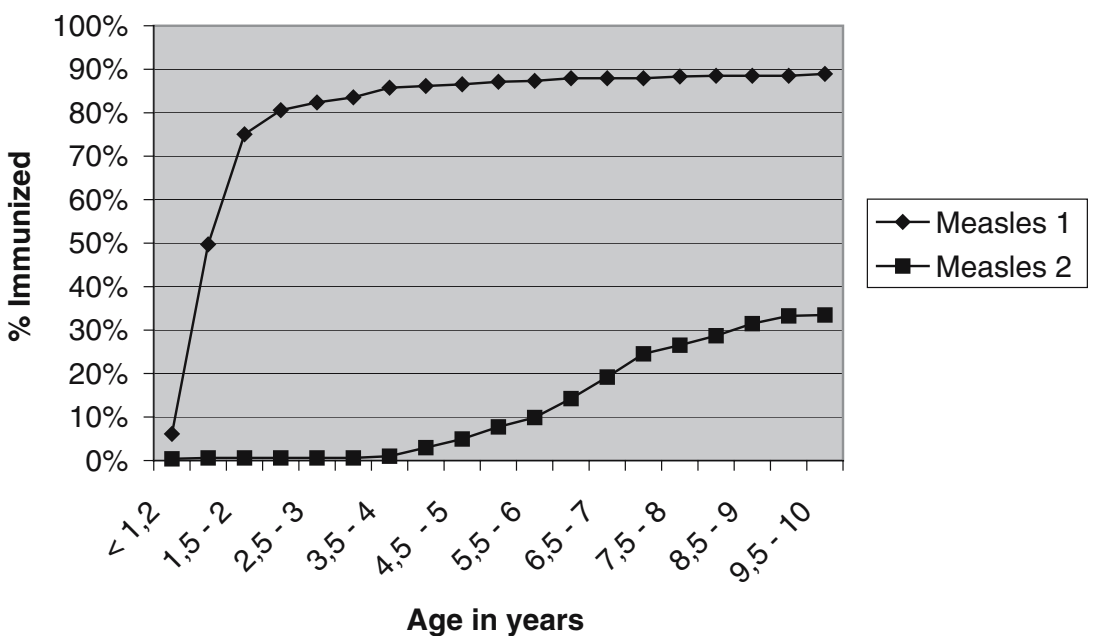

for reasons as stated in Fig. 1. The remaining 236 kindergarten children were 5.2 years old on average (interquartile range: 4.7-6.4) and the 269 school-aged children were 9.4 years of age (inter-quartile range: 8.5-10.7). There were 241 girls $(47.7 \%)$ and 264 boys.

\section{Immunization coverage}

The overall immunization rates are demonstrated in Table 1. As can be seen, coverage for the primary series (three doses) was $>95 \%$ for diphtheria, tetanus, and poliomyelitis and $<90 \%$ for pertussis and Hib. However, the rates of recommended booster doses were significantly lower, especially for pertussis and Hib. Specifically, coverage for the fifth dose of pertussis was disappointingly low. Also, coverage for MMR was below $90 \%$ for the first dose in the total study cohort and approximately $50 \%$ for the second dose in children 7 years of age or older at the time of the study.

\section{Timely administration of immunization}

For the assessment of timely immunization, the following analysis was restricted to 304 children born after 31 December 1991, when the recommended time points for DTP were changed from 2-3-4 to 2-4-6 months of age. In Fig. 2, cumulative coverage of the first three doses of diphtheria immunization is demonstrated to exemplify vaccination with DTP combination vaccines. As can be seen, the $90 \%$ threshold was reached with significant delay, i.e., at 110 days, 205 days, and 370 days for doses 1-3.

A forth dose of pertussis vaccine to be administered at 15-24 months of age was introduced in Switzerland in January 1996. To assess compliance and timely administration of this recommendation, the cumulative vaccination coverage of children who were 15 months of age or older in January 1996 is demonstrated in Fig. 3 in comparison to the total study cohort. By 2 years of age, approximately $65 \%$ of young children had received a forth dose, but a plateau is reached just below $80 \%$ at 4 years of age. This indicates not only suboptimal overall coverage, but also delayed administration in those who are immunized at all. Furthermore, compliance with the forth dose was also analyzed in the total study cohort, because the forth dose has been recommended as a catch-up immunization in children older than 15-24 months of age. By 10 years of age, $55 \%$ of all study children had received the reinforcing forth dose.

Finally, cumulative coverage with two doses of measles containing vaccine (exemplary for MMR) was evaluated (Fig. 4). By 2 years of age, the end of the recommended time period for the first dose, $75 \%$ had been immunized once. Coverage increased gradually to $82 \%, 86 \%$, and $88 \%$ at 3,4 , and 7 years (school entry) of age, respectively, but remained below $90 \%$ throughout all the age groups investigated in this study. Administration of the second dose was even more retarded: by 7 years of age, the end of the recommended time period relevant for children included in this study, 19\% had received a second dose of measles containing vaccines. The coverage for the two doses of measles vaccines was $33 \%$ in the total study cohort (Fig. 4), whereas in children 7 years of age or older, the rate was approximately $50 \%$ (Table 1 ).

\section{Discussion}

In this study, we prospectively assessed immunization coverage and - for the first time in Switzerland-timely administration of generally recommended vaccinations in a large cohort of kindergarten and school-aged children. Overall, coverage for the primary series of immunizations against diphtheria, tetanus, and poliomyelitis was excellent (above 95\%) and was acceptable (close to 90\%) for pertussis and Hib. However, coverage rates for booster doses were significantly lower, which is of great concern, given that vaccine-induced immunity will wane over time [20]. Furthermore, coverage rates for the first and second dose of MMR were below the targets established by the 
WHO [21]. Unfortunately, these findings are in line with previous surveys performed in various areas of Switzerland and elsewhere in Europe, and jeopardize the goal of the elimination of measles and congenital rubella syndrome by the year 2010 [21]. Reasons for less than optimal immunization rates in a number of western European countries are numerous, and further efforts such as reminder recall systems, education of professionals and the public, as well as providing more opportunities for vaccination are needed for improvement [2].

Data on timely administration of recommended childhood immunizations are rare, especially in Europe [3, 8]. In a study performed in Germany in 1999 , only $40-56 \%$ of children had received their primary series of immunizations against DTP, Hib, polio, and hepatitis B [8]. At 24 months of age, the rates were in the range of $73-90 \%$ and $53-62 \%$ for primary and booster immunizations, respectively. With regards to MMR, $73 \%$ of children had received their first dose by 2 years of age.

A retrospective cohort study among children who entered public schools in Chicago, USA, in 2001 and 2002 revealed that only $31 \%$ of children had received all of the recommended 2-4-6 month immunizations by 7 months of age, and the percentage increased to merely $64 \%$ at 13 months of age [5]. With regards to administration of the first dose of measles containing vaccine, the initial delay was also significant, although by the time of school entry, $97 \%$ were immunized. The authors concluded that striking immunization delays exist, which require more attention. Similar results were obtained from the 2003 National Immunization Survey in the USA, which was designed to estimate vaccination coverage for children age 19-35 months [10]. In children up to 24 months of age, the cumulative mean delay of recommended immunizations was 172 days, i.e., 6 months. Specifically, 9\% (regarding polio) to $21 \%$ (regarding $\mathrm{Hib}$ ) of all children had delays of more than 6 months. The authors postulated that greater emphasis should be put on minimizing the time spent incompletely protected from vaccine-preventable diseases, especially in young children.

In comparison, our findings are similarly worrisome and indicate a need for action. With regards to the primary series of DTP, approximately $65 \%$ of children were completely immunized by 7 months of age, and so were $90 \%$ by 1 year of age (Fig. 2). By 3.5 years of age, 18 months after the latest recommended time point, coverage for the forth dose of pertussis reached a plateau at approximately $80 \%$ in children born from September 1994 onwards. Of concern, coverage for the four doses was below $60 \%$ for the total study population. This indicates that many physicians do not comply with the national recommendation for catch-up immunizations against pertussis in older children and, therefore, herd immunity in the community can not be expected. Not surprisingly, pertussis epidemics continue to occur in school-aged children in Switzerland [11].

With regards to measles, usually given as MMR, $75 \%$ of children had received their first dose by 2 years of age, and coverage only increased slowly thereafter to reach $89 \%$ by 10 years of age. Again, catch-up immunizations were rarely applied after the recommended age for the first dose, i.e., the second year of life. Importantly, the concept of optimal protection by use of a two-dose schedule for MMR has not been implemented at a high level in our area. At school entry, only $20 \%$ of children had received a second dose and the rate did not exceed $33 \%$ by 10 years of age.

Our study has some limitations. First and importantly, our study design did not allow us to assess the reasons for delay or complete lack of specific immunizations. Previous studies in other communities have revealed several factors which contribute to under-immunization. On the health care provider side, these include missed opportunities for immunization, misconceptions about the importance of timely immunization, inappropriate contraindications, and lack of recall systems to identify under-immunized children [18]. On the family side, refusal of specific vaccinations, birth order (second or later born), less educated mother, and one-parent-family status have been identified as risk factors for under-immunization in children $[1,9,15,17]$.

Second, the assessment of immunization status relied on written documentation in a child's official immunization booklet. No efforts were made to verify how accurate these documents were. Therefore, we may have misclassified some children as not immunized when, in fact, the respective vaccination had been performed but not documented. Although vaccination coverage, therefore, might have been underestimated, this would not create a bias for analyses of delayed administration.

Third, about $18 \%$ of the potential study children were excluded from analyses because they were either of foreign birth or no immunization records were available, either because of parental refusal to participate or due to loss of records. Unfortunately, the design of our study did not allow us to discriminate "refusal to participate" and "loss of documents" as reasons for exclusion, as this question had not been asked formally. For this reason, we do not know if and to which direction this might have biased our findings. In our daily experience, foreign-born children have higher or lower immunization rates than Swiss children, depending on their country of origin. In contrast, the children of parents who hesitate to reveal immunization records frequently have immunization gaps. Therefore, our estimates on vaccination coverage may be too optimistic, but again, we do not think that there would be a strong bias on the overall findings of delayed immunizations.

In conclusion, we provide evidence for less than optimal coverage of and significant delays in generally recommended immunizations in one specific area of Switzerland. We have no reason to believe that the situation would be significantly different in other parts of the country. This is of great concern and has important implications for public health. Specifically, the timely start of vaccination is important in light of the rapid waning of transplacental immunity (e.g., against measles) in the first year of life and major health threats for infants caused by vaccine-preventable infectious diseases, such as pertussis and invasive Hib disease [7, 19]. Further, delayed or even completely missing booster doses puts individuals at unnecessary risk of disease due to waning immunity over time and puts the 
whole community at risk of epidemics [6]. Greater efforts are needed to decrease the duration of nonprotection caused by incomplete and delayed immunizations.

Acknowledgements We are grateful to Dr. Iris Meier, School Health Service of the Canton of Basel, Switzerland, for her assistance and support in conducting this survey. Further, the willingness of the parents who participated is gratefully acknowledged.

\section{References}

1. Bobo JK, Gale JL, Thapa PB, Wassilak SG (1993) Risk factors for delayed immunization in a random sample of 1163 children from Oregon and Washington. Pediatrics 91:308-314

2. Briss PA, Rodewald LE, Hinman AR, Shefer AM, Strikas RA, Bernier RR, Carande-Kulis VG, Yusuf HR, Ndiaye SM, Williams SM (2000) Reviews of evidence regarding interventions to improve vaccination coverage in children, adolescents, and adults. The Task Force on Community Preventive Services. Am J Prev Med 18(Suppl):97-140

3. CDSC (2000) COVER programme: October to December 1999 vaccination coverage statistics for children up to 5 years of age in the United Kingdom. Commun Dis Rep CDR Wkly 10:109110

4. Dombkowski KJ, Lantz PM, Freed GL (2004) Risk factors for delay in age-appropriate vaccination. Public Health Rep 119:144-155

5. Dominguez SR, Parrott JS, Lauderdale DS, Daum RS (2004) On-time immunization rates among children who enter Chicago public schools. Pediatrics 114:e741-e747

6. Grimprel E, Baron S, Levy-Bruhl D, Garnier JM, N'jamkepo E, Guiso N, Begue P (1999) Influence of vaccination coverage on pertussis transmission in France. Lancet 354:1699-1700

7. Heininger U, Stehr K, Cherry JD (1992) Serious pertussis overlooked in infants. Eur J Pediatr 151:342-343

8. Laubereau B, Hermann M, Weil J, Schmitt HJ, von Kries R (1999) Durchimpfungsraten bei Kindern in Deutschland 1999. Monschr Kinderheilkd 149:367-372
9. Li J, Taylor B (1993) Factors affecting uptake of measles, mumps, and rubella immunisation. BMJ 307:168-171

10. Luman ET, Barker LE, Shaw KM, McCauley MM, Buehler JW, Pickering LK (2005) Timeliness of childhood vaccinations in the United States: days undervaccinated and number of vaccines delayed. JAMA 293:1204-1211

11. Matter HC (1999) Pertussis surveillance in Switzerland, 1992 to 1997 - a large epidemic in 1994. What next? Euro Surveill $4: 130-133$

12. Maurer AM, Muhlemann K, Seiler AJ (2001) Vaccination of young children, at school entrance and completion in the Bern canton 1998 (in German). Schweiz Rundsch Med Prax 90:1676-1684

13. Paget JW, Zimmermann H, Vorkauf H (2000) A national measles epidemic in Switzerland in 1997: consequences for the elimination of measles by the year 2007. Euro Surveill 5:17-20

14. Roth-Kleiner M, Gnehm HE (1997) MMR, diphtheria-tetanus and polio vaccination of students in Aargau. Schweiz Rundsch Med Prax 86:1934-1937

15. Schaffer SJ, Szilagyi PG (1995) Immunization status and birth order. Arch Pediatr Adolesc Med 149:792-797

16. Siegrist CA (2005) Vaccinology update 2004 (in French). Rev Med Suisse 1:79-82

17. Szilagyi PG, Rodewald LE, Humiston SG, Raubertas RF, Cove LA, Doane CB, Lind PH, Tobin MS, Roghmann KJ, Hall CB (1993) Missed opportunities for childhood vaccinations in office practices and the effect on vaccination status. Pediatrics 91:1-7. Erratum in: Pediatrics 91:545

18. Szilagyi PG, Rodewald LE (1996) Missed opportunities for immunizations: a review of the evidence. J Public Health Manag Pract 2:18-25

19. von Kries R, Bohm O, Windfuhr A (1997) Haemophilus influenzae b-vaccination: the urgency for timely vaccination. Eur J Pediatr 156:282-287

20. Wendelboe AM, Van Rie A, Salmaso S, Englund JA (2005) Duration of immunity against pertussis after natural infection or vaccination. Pediatr Infect Dis J 24:S58-S61

21. World Health Organization (2005) Progress towards elimination of measles and prevention of congenital rubella infection in the WHO European Region, 1990-2004. Wkly Epidemiol Rec 80:66-71 